\title{
El liderazgo del rectorado en los procesos de evaluación con fines de acreditación internacional
}

\author{
The leadership of the rector in the assessment processes for international accreditation
}

Recibido 05 junio 2015 • Aceptado 14 setiembre 2015 • Corregido 05 octubre 2015

\author{
Magdalena Liliana Bustos Aguirre ${ }^{1}$ \\ Departamento de Turismo, Recreación y Servicio \\ Universidad de Guadalajara, Jalisco, México \\ magda.bustos@gmail.com
}

Rosa Vega Cano ${ }^{2}$

Facultad de Economía

Universidad Michoacana de San Nicolás de Hidalgo, Michoacán, México

rosavc@yahoo.com.mx

Diana Rojo Morales ${ }^{3}$

Departamento de Cultura, Arte y Desarrollo Humano

Universidad de Guadalajara, Jalisco, México

diana.rojo01@gmail.com

Resumen. El presente ensayo destaca el papel del rectorado en los procesos de evaluación y acreditación internacional de las instituciones de educación superior, pues su visión e involucramiento pueden contribuir a mantener el enfoque del proceso como medio para fomentar la calidad en la institución. Sin entrar en el debate que ha implicado la inclusión del concepto de calidad educativa, este trabajo trata de responder a la siguiente interrogante: ¿qué estilo de liderazgo deberá ejercer el rector o rectora de una institución de educación superior que se embarca en proceso de evaluación o acreditación internacional? El trabajo concluye que el liderazgo del rectorado es una piedra angular en el establecimiento de una cultura de calidad en las instituciones de educación superior, así como

1 Profesora de tiempo completo de la Universidad de Guadalajara, adscrita al Departamento de Turismo, Recreación y Servicio. Actualmente es estudiante del Doctorado en Gestión de la Educación Superior de la Universidad de Guadalajara.

2 Profesora-Investigadora de la Universidad Michoacana de San Nicolás de Hidalgo, adscrita a la Facultad de Economía. Actualmente es estudiante del Doctorado en Gestión de la Educación Superior de la Universidad de Guadalajara.

3 Profesora de tiempo completo de la Universidad de Guadalajara, adscrita al Departamento de Cultura, Arte y Desarrollo Humano. Actualmente es estudiante del Doctorado en Gestión de la Educación Superior de la Universidad de Guadalajara. 
en los procesos de evaluación y acreditación internacional, y señala que los estilos de liderazgo más importantes en estos procesos son: el liderazgo transformacional, el liderazgo trascendente y el liderazgo transaccional.

Palabras clave. Gestión de la educación superior; evaluación de la educación superior; instituciones educativas acreditadas; planificación de la educación

\begin{abstract}
The present essay addresses the role of the rector in the evaluation and accreditation processes at higher education institutions, since it is the rector's vision and involvement which can contribute to maintain the focus of the process as a means to increase the quality of the institution. Without entering into the debate on what quality means in the education arena, this essay will try to answer the following question: what leadership styles should a rector of a higher education institution exercise when embarking in an international evaluation and accreditation process? This essay concludes that the leadership of the rector is a cornerstone when stablishing a culture of quality within the higher education institution and when embarking in international evaluation and accreditation processes, and highlights that the most important leadership styles in these processes are: transformational leadership, transcendental leadership and transactional leadership.
\end{abstract}

Keywords. Higher education management; evaluation in higher education; accredited education institutions; planning in education

\title{
Introducción
}

A partir del fenómeno de la masificación que inició en la década de los setenta, comenzó una preocupación generalizada por la calidad en la Educación Superior, pues las condiciones vigentes de las Instituciones de Educación Superior (IES) para atender a una élite resultaron claramente insuficientes para dar cabida a las grandes cantidades de jóvenes que comenzaron a inundar sus aulas. Esta preocupación dio lugar, a partir de la década de 1990, a la implementación, en prácticamente todos los países, de sistemas de aseguramiento de la calidad y de un posicionamiento del tema en el centro del debate durante los últimos cinco lustros. No obstante, al no existir una definición consensuada de lo que significa calidad en términos de Educación Superior, se optó, en términos de políticas, por abordar el tema mediante la regulación y estandarización de ciertas prácticas que según el consenso público podrían considerarse como indicativas de calidad, estas pasaron a formar parte de los procesos de evaluación y acreditación (Blanco-Ramírez y Berger, 2014) y que condujeron a que las evaluaciones y acreditaciones internacionales de programas y de instituciones se constituyeran como garantes de la calidad de una IES.

Por otra parte, el fenómeno de la globalización -el cual se define como una serie de procesos que evidencian el cambio en la organización espacial de las relaciones y transacciones sociales, generando flujos y redes transcontinentales e interregionales de actividad, interacción 
y ejercicio del poder, que dan lugar al ensanchamiento, intensificación, aceleración y creciente impacto de la interconexión mundial (Held, McGrew, Goldblatt y Perraton, 2000), y que trascendió el ámbito económico que la vio nacer para posicionarse en prácticamente todas las esferas de la vida- ha reiterado la preocupación por el mejoramiento de la calidad en las IES (Botero Chica, 2008) y lo ha internacionalizado.

El fenómeno que ha conducido a que la evaluación y acreditación internacional de las IES se difunda en todo el mundo es la internacionalización, esta se define como todos aquellos esfuerzos que las IES emprenden para adaptarse a las nuevas condiciones económicas, políticas y sociales de la globalización (Van der Wende, 1997) desde la propia idiosincrasia e identidad nacional. Dado que la internacionalización de la Educación Superior implica también una interacción constante entre las IES de diferentes países, existe una demanda cada vez mayor de mecanismos que permitan la comparabilidad en términos de calidad entre las instituciones del orbe.

Los procesos de aseguramiento de la calidad al no pertenecer únicamente a un país, son internacionales en su naturaleza y constituyen también un ejemplo de internacionalización; de igual modo, las prácticas para asegurar la calidad y las políticas que les dan sustento se transfieren de una institución a otra, de una región a otra y de un país a otro. Según BlancoRamírez y Berger (2014), esto es resultado de la adopción activa de prácticas de calidad de otros entornos, de atender las recomendaciones de organismos internacionales en la materia y de que muchos países e instituciones invitan a expertos internacionales a contribuir en el diseño de sus esquemas de aseguramiento de la calidad.

Esta internacionalización de los esquemas de aseguramiento de la calidad también se percibe al estudiar las semejanzas entre los diferentes modelos existentes en el mundo, pues la mayoría están basados en el modelo que Van Vaught y Westernheijden desarrollaron en 1993 y tienen los siguientes aspectos: estándares similares, revisión por pares como elemento central del proceso, y uso de formatos parecidos para documentar los resultados de la calidad (BlancoRamírez y Berger, 2014).

Este proceso de adopción de prácticas de calidad se ha dado paulatinamente, a través del vencimiento de resistencias y de forjar una nueva cultura organizacional, y no de forma automática, particularmente en los países donde los procesos de aseguramiento de la calidad no son coercitivos. Implica un cambio organizacional en pro de una cultura de evaluación externa que requiere de un liderazgo decidido, asertivo y promotor del cambio al interior de la institución.

Normalmente no es un proceso libre de debate, pues muchas voces se levantan en contra de las prácticas de aseguramiento de la calidad y se cuestiona intensamente si realmente contribuyen a elevar los niveles de calidad de las funciones sustantivas de las IES. Este debate indica que, al parecer, las instituciones y sus actores han soslayado la importancia de la evaluación como un mecanismo que propicia la reflexión y el análisis de las prácticas académicas, y han olvidado que los mecanismos de evaluación no son un fin en sí mismos, sino 
un medio para diagnosticar la pertinencia de la propia institución. En este contexto, la rectoría de una institución debe asumir un papel de agente de cambio, pues su visión e involucramiento pueden contribuir a mantener el entusiasmo y el enfoque, recordando a los integrantes de la comunidad académica que la evaluación es un medio de reflexión sobre las prácticas y los resultados hasta ahora obtenidos, el cual sirve como un referente para la planeación estratégica, la definición y redefinición de objetivos y metas.

De igual forma, es importante recordar que no hay consenso sobre lo que para la Educación Superior significa calidad, aun cuando sí lo puede haber para lo que se entiende por mejores prácticas, y en cuanto a los métodos, estándares y proxis involucrados en la evaluación y la acreditación; en este sentido y dada la variedad de perspectivas sobre la calidad y la falta de una definición consensuada y coherente del término, parece más sensato hablar de procesos a través de los cuales la calidad se construye y promulga en las IES (Blanco-Ramírez y Berger, 2014), definición a la que este documento se adhiere.

Considerando lo anterior, este trabajo pretende responder la siguiente pregunta: ¿qué estilo de liderazgo deberá ejercer el rector o rectora de una IES que se embarca en el proceso de evaluación o acreditación internacional?

En cuanto a su metodología, el presente ensayo es de tipo conceptual y teórico, pues se basa en una revisión de la literatura existente sobre el tema aquíabordado. Está dividido en cuatro apartados: comienza con una revisión teórica sobre el liderazgo y sus tipos, posteriormente se analizan la evaluación y acreditación internacionales con la finalidad de entender su génesis y los desafíos que para las IES implica, después se analizan las implicaciones de los procesos de evaluación y acreditación internacional, y finalmente se establecen algunas conclusiones.

\section{El liderazgo}

El concepto de liderazgo ha sido ampliamente abordado desde diferentes disciplinas y autores y existe una variedad de formas de concebirlo. Esta diversidad de acepciones implica que, al hacer referencia al término, se deben tener en cuenta múltiples dimensiones y enfoques, pues es un concepto que contiene elementos de tipo político, filosófico y sociológico. Casares (1994), citado por Madrigal (2013), sostiene que:

[Liderazgo] es la acción de influir en los demás; las actitudes, conductas y habilidades de dirigir, orientar, motivar, vincular, integrar y optimizar el quehacer de las personas y grupos para lograr los objetivos deseados, en virtud de su posición en la estructura de poder y promover el desarrollo de sus integrantes. (p. 34)

Independientemente del autor, el enfoque o la disciplina desde la cual se aborde el tema, parece existir coincidencia en que el liderazgo consta de cuatro componentes: 
1) La capacidad para hacer un uso eficaz y responsable del poder, 2) la capacidad para comprender que los seres humanos tienen diferentes motivaciones en diferentes momentos y situaciones, 3) la capacidad para inspirar a los demás y 4) la capacidad para actuar a favor del desarrollo de una atmósfera conducente a la respuesta ante las motivaciones y al surgimiento de estas. (Koontz y Weihrich, 1998, p. 533)

Así como existen diferentes enfoques y acepciones, existen también distintos estilos de liderazgo. Madrigal (2013) asevera que dos orientaciones básicas definen los estilos de liderazgo: la orientación a la tarea y la orientación a las relaciones. Esto está en coincidencia con la teoría de la contingencia de Fiedler, quien propone que el desempeño eficaz de un equipo depende del estilo de interacción del líder con sus subordinados y de la forma en la que se desenvuelve esta interacción, considerando su orientación a las personas y a la tarea. Esta teoría toma en cuenta tres elementos básicos: las características del líder, su efectividad y el control de la situación (Robbins, 2004). Así, los líderes orientados a la tarea fomentan altos niveles de productividad y definen las actividades de su equipo de trabajo en función de objetivos, mientras que el líder orientado a las personas está mucho más interesado en las necesidades, interrelaciones, problemas y desarrollo de sus colaboradores que en la tarea, las personas son primero y por tanto su actuación está encaminada a satisfacer a sus colaboradores y lograr su desarrollo antes que a cumplir los objetivos de la empresa o tener altos estándares de desempeño.

Madrigal (2013) también sostiene que, un líder que apenas emprende la tarea de coordinar un grupo de trabajo, podría tener como referente cuatro estilos básicos de liderazgo: el estructurador, el entrenador, el alentador y el delegador; esta autora también sugiere que, conforme el nuevo líder avance en la consolidación de su equipo, debe desplazarse de un estilo de liderazgo al siguiente. Las características de estos estilos son:

- Liderazgo estructurador:el líder decide y comunica la tareay los subordinados ejecutan. En general los subordinados poseen conocimientos o experiencia mínima. No debe usarse con personas experimentadas pues puede llevarles a dejar de cooperar.

- Liderazgo entrenador: se emplea con personas que tienen interés en aprender y potencial para hacerlo. La relación líder - subordinado(a) tiene dos fines, cumplir la tarea y desarrollar las habilidades y la responsabilidad necesarias en el colaborador. Es un estilo que demanda mucho compromiso y energía por parte del líder.

- Liderazgo alentador: busca aumentar la confianza y habilidad en las personas que lidera al reconocer sus logros y dejarlos decidir y solucionar problemas. Este estilo de liderazgo solo puede ser ejercido con personas que se desempeñan por encima del estándar.

- Liderazgo delegador: la persona que lidera asigna tareas adecuadamente, verificando, supervisandoy direccionando ocasionalmente. Se asegura de que sus subordinados(as) 
cuenten con los recursos necesarios para realizar su tarea y les permite establecer sus propias metas. La tarea principal del líder delegador es asegurar que se alcancen las metas, se sigan las políticas y que no existan interrupciones.

Ahora bien, es importante recordar que no existe un estilo ideal único de liderazgo que pueda ser asumido por quien tenga a su cargo la dirección de una IES, pues un líder debe actuar y reaccionar ante una serie de estímulos y situaciones contingentes que le demandarán utilizar diferentes estilos de liderazgo e intercambiarlos flexiblemente, tomando en cuenta el tipo de organización que dirige, así como la cultura, características y personalidades de sus integrantes. Lo más importante, sin embargo, será que el líder conozca las motivaciones de su equipo de trabajo para poner en juego un estilo de liderazgo que conduzca a la organización, como un todo, a lograr altos estándares de desempeño en el cumplimiento de las metas y objetivos establecidos.

El siglo XXI, marcado por la globalización y el uso de las tecnologías de la información y las comunicaciones, demanda líderes que conduzcan procesos compartidos, que dialoguen, canalicen y potencien la confluencia de voces múltiples y diversas, que inspiren y estimulen, que fortalezcan el encuentro de opciones múltiples, que sean capaces de tener una visión a futuro de sus organizaciones y que sean proactivos. Algunos autores, como Madrigal (2013) y Bolívar (1997), indican que los tipos de liderazgos que se requieren para el siglo XXI son:

a) El liderazgo transformacional o transformador, es aquel que desarrolla una visión, motiva, emociona e inspira para cumplir objetivos, estimula intelectualmente y proporciona nuevas ideas y enfoques; también pone atención en los intereses y necesidades de desarrollo de sus seguidores, espolea y retiene el talento, es carismático y visionario, considera las características y necesidades individuales de sus colaboradores, tolera bien la frustración, es capaz de construir un liderazgo compartido y trabaja en equipo, cuida su formación continua y la de sus subordinados(as), sin olvidarse de que desempeña el papel de autoridad; se trata también de un estilo de liderazgo que implica un enfoque individualizado.

b) El liderazgo trascendente busca, no solo realizar transacciones en su contexto, sino orientar y transformar su espacio de acción impactando con ello el contexto institucional. Es un liderazgo que inspira y se compromete con sus seguidores, que es dinámico y que alcanza una vinculación ética con su equipo de trabajo, logrando que estos interactúen con su líder por motivaciones extrínsecas, intrínsecas y trascendentes. Se basa en propósitos institucionales compartidos y en el apoyo de los miembros de la institución; requiere una estructura administrativa y académica descentralizada que motive intelectualmente a tener un mejor desempeño profesional y que promueva una cultura de colaboración.

c) El liderazgo relacional, implica la existencia de líderes capaces de discernir, con visión sistémica, que propicien relaciones interpersonales, que lideren el cambio y formen 
nuevos líderes, que aprendan a desaprender para construir nuevos modos deconsiderar la vida, y que utilicen como sus herramientas más importantes la empatía, la sinergia, la comunicación, la observación, la escucha atenta y la gestión del conocimiento.

Bolívar (1997) menciona también al liderazgo transaccional como útil en la gestión escolar, pues está basado en el intercambio de recompensas entre quien ejerce el liderazgo y los miembros de su equipo de acuerdo con el cumplimiento de objetivos. Este liderazgo puede ser 'activo', cuando la desviación de las reglas obliga a corregir acciones, o 'pasivo', interviniendo solo si el surgimiento de problemas no permite el cumplimiento de objetivos. Los integrantes del equipo realizan su labor y a cambio sus refuerzos son recompensados por su líder. Cuando este tipo de liderazgo es bien aplicado se consiguen efectos positivos en el ánimo y desempeño de los colaboradores y las colaboradoras.

Ahora bien, planear cambios en la institución con la finalidad de hacer frente a los retos que supone la modernidad educativa, considerando que la acreditación internacional es uno de estos, implica no solamente dirigir a la comunidad universitaria, sino también "crear roles y estructuras que apoyen y promuevan las prácticas educativas que deseamos" (Sikes y Elmore, 1989, citados por Bolívar, 1997, p. 27). Garantizar el éxito en los procesos de cambio organizacional para dar cabida a los procesos de aseguramiento de la calidad en general, y de la evaluación y acreditación internacional en particular, implica una respuesta adaptativa en la que los líderes de las IES no solamente deben ser gestores del cambio, sino sus principales promotores, facilitando, siempre que sea posible, que sus colaboradoras y colaboradores reciban aquellas recompensas que consideran valiosas; creando, unificando, desarrollando y difundiendo las comunicaciones necesarias al interior y exterior de la IES; optimizando recursos y utilizando todas las herramientas de gestión disponibles para facilitar los procesos de análisis y toma de decisiones; e implementando una cultura que favorezca el aprendizaje continuo y que ponga énfasis en el desarrollo de competencias en los integrantes del equipo (Palomino Vadillo, 2013).

Abonando al debate, Uribe (2007) refiere que las prácticas de gestión revelan en gran medida el nivel de competencias alcanzadas por quienes ostentan los cargos de dirección, lo que indica la necesidad de una construcción social de aprendizajes significativos y útiles para el desempeño de la dirección institucional. Aunque se reconoce que, en gran medida, este aprendizaje lo obtienen las autoridades escolares a partir de sus experiencias, es evidente que se derivan también de la combinación de recursos cognitivos y no cognitivos, así como de la ejecución de saberes conceptuales, procedimentales y actitudinales, permitiendo a quien dirige una institución responder exitosamente a las complejas exigencias de las IES.

Ahora bien, de acuerdo con Raczynski (2005), citado por Uribe (2007), quienes asumen los cargos de dirección de manera efectiva logran crear un clima organizacional de apoyo a las diferentes actividades escolares, e incluyen al personal docente en la toma de decisiones, planifican en conjunto y monitorean las actividades pedagógicas, no por control, sino para 
brindar apoyo. Por el contrario, las personas que asumen los cargos de dirección con un estilo de liderazgo poco efectivo no apoyan las actividades ni al personal docente, no promueven la definición de objetivos en su institución, y fomentan el surgimiento de acciones individuales aisladas en detrimento de la propia IES.

En resumen, se puede decir que el rectorado y su equipo de trabajo son clave en la definición de estrategias de mejoramiento de las IES y que su estilo de liderazgo marcará la tendencia y actitudes del resto de la comunidad universitaria que participe en el proceso, así como el éxito de la encomienda. De igual manera, las exigencias de la nueva gestión pública, donde se insertan los esquemas de aseguramiento de la calidad, requieren un nuevo perfil en quienes ejercen la rectoría de las IES, en palabras de Acosta Silva (2010, p. 17), se requieren autoridades híbridas, que combinen "las capacidades del político (El Príncipe de Maquiavelo), con el expertise del burócrata profesional o de sus consejeros de ocasión (fiel al modelo weberiano), con las del gerente de las políticas institucionales (la figura predilecta en el campo de la Nueva Gestión Pública)".

\section{Evaluación y acreditación internacional}

Como ya se mencionó, no hay duda de que el tema del aseguramiento de la calidad ha sido un tema importante en Educación Superior durante más de dos décadas: como un asunto de políticas, como un enfoque de práctica y como un área de estudio e investigación. Las prácticas de calidad no simplemente suceden, son desarrolladas a través del involucramiento activo de agentes institucionales, tanto al interior como al exterior de un país, y son generalmente adoptadas por la legitimidad que se cree implican, independientemente de las diferencias contextuales y sociales entre países (Blanco-Ramírez y Berger, 2014). Así, el aseguramiento de la calidad se ha erigido como un elemento de diferenciación y posicionamiento en el mercado de la Educación Superior y, a su vez, ha generado la definición de estándares comunes de evaluación y acreditación para la supervisión y regulación de la calidad de la educación que las IES ofrecen.

La evaluación puede ser entendida como un proceso ordenado continuo y sistemático de recogida de información, cuantitativa y cualitativa, obtenida a través de ciertas técnicas e instrumentos que, tras ser cotejada o comparada con los criterios establecidos, permite emitir juicios de valor fundamentados que ayudan al proceso de toma de decisiones sobre el objeto evaluado (Jiménez, 1999). La acreditación, por su parte, "debe ser considerada como el método para evaluar la calidad y la pertinencia de una institución o programa determinado de educación superior, permitiendo que sea reconocida dentro de un sistema de educación superior" (Global University Network for Innovation [GUNI], 2009, p. 163). Evaluación y acreditación se convierten, entonces, en un binomio difícil de separar en un contexto caracterizado por la competencia entre IES.

Viñas (1991) señala que la evaluación con fines de acreditación tuvo su génesis y desarrollo en Estados Unidos y surgió para certificar las competencias profesionales de organizaciones prestadoras de servicios de salud y, posteriormente, se adaptó el proceso para la acreditación de 
IES. Tanto la evaluación como la acreditación se llevan a cabo en distintos niveles: institucional, de programas educativos y de sistemas de Educación Superior.

De acuerdo con Sanyal y Martin (2009) la acreditación es uno de los métodos de garantía externa de la calidad más empleados en el mundo educativo, lo que se explica a partir de cinco elementos: la existencia de un mayor número de proveedores de Educación Superior; un mayor nivel de competencia entre IES; la existencia de una mayor diversificación de la oferta de educación; un crecimiento en la demanda por más y mejor educación; y la garantía que proporciona un título universitario expedido por una escuela acreditada.

Poco a poco, la lógica del occidente capitalista sobre la productividad y la eficiencia fue dominando el escenario de la Educación Superior y generando una competencia cada vez más compleja entre las IES. Esto, a su vez, dio lugar a una evolución de los lineamientos de evaluación y acreditación, de tal forma que transitaron de la evaluación cuantitativa a una cualitativa; de la evaluación de insumos a la de resultados; de la evaluación con base en las misiones institucionales a la evaluación con base en parámetros de calidad predefinidos; de la preeminencia de la autoevaluación a una mayor valoración de la evaluación externa; de la evaluación centralizada a una evaluación regionalizada; de la evaluación académica a la de múltiples dimensiones; de la evaluación para el mejoramiento de la calidad a la evaluación para la obtención de recursos presupuestales; de las evaluaciones de conocimientos a las evaluaciones de competencias profesionales; $y$ de las evaluaciones presenciales a evaluaciones a través de sistemas informáticos (Rama, 2009).

Los procesos de evaluación y acreditación incorporaron la dimensión internacional a partir de la Conferencia Mundial sobre la Educación Superior de la Organización de las Naciones Unidas para la Educación, la Ciencia y la Cultura (UNESCO), en 1998 (GUNI, 2009), y se intensificaron con la transnacionalización de la Educación Superior, pues las IES, al traspasar sus fronteras e insertarse en otros países, comenzaron a someterse a presiones que les llevaron a buscar la acreditación local e internacional como un medio para legitimarse (Knight y De Wit, 2007). Por tanto, la acreditación internacional puede ser entendida como la convergencia de las agencias nacionales sobre criterios comunes y reconocimiento mutuo de sus respectivas acreditaciones y como un valor añadido al proceso educativo que permite mayores niveles de comparabilidad entre las IES y de movilidad internacional de los profesionales de un país (Rama, 2012).

La demanda de procesos de acreditación internacional generó que agencias de calidad de diferentes países se embarcaran en prácticas de internacionalización de la acreditación y se fundamentó en la necesidad de construir procesos de regulación internacional que tuvieran elementos comunes de evaluación y acreditación como parte de la estandarización que caracteriza a la globalización.

No obstante, la inserción de la dimensión internacional en la acreditación de las IES complejiza el proceso, pues implica la incorporación de una multiplicidad de elementos de 
diversa índole: jurídico, administrativo, geográfico, político, económico y cultural, considerados todos ellos en la escala supranacional. Sebastián (2003) señala que existen cuatro ámbitos en los que se puede analizar la problemática por la internacionalización de la acreditación: en los organismos evaluadores y acreditadores, los cuales han rebasado la esfera nacional y han incorporado estándares y prácticas de otras latitudes; en los objetos de la evaluación, es decir, en las instituciones, los programas de estudio, el personal docente y el alumnado incluidas las generaciones ya egresadas, donde se miden temas centrales relacionados con la internacionalización de la docencia, la investigación y la difusión, tales como publicaciones, movilidad estudiantil y programas conjuntos; en los métodos y procedimientos de la evaluación, los cuales toman de referencia las buenas prácticas internacionales y las recomendaciones de organismos internacionales; y en los productos de la evaluación, que se refieren a las certificaciones y acreditaciones que tienen valor más allá de las fronteras.

Por su parte, la intención de adoptar esquemas internacionales para el aseguramiento de la calidad en las IES se vincula con motivos de diversa índole: económica, pues contar con este tipo de distintivos contribuye a la consecución de recursos extraordinarios; académica, pues implica lograr estándares internacionales en docencia e investigación; institucional, pues las acreditaciones internacionales también contribuyen a mejorar la reputación y perfil internacional de la institución; y de competencia internacional, dado que la venta de servicios educativos en otros países es una tendencia cada vez más posicionada en el escenario de la Educación Superior.

Independientemente de los motivos que sustenten, la búsqueda de acreditaciones internacionales por parte de las IES, no es un tema en el que haya un consenso generalizado: por una parte se cuestiona la relación entre calidad y acreditación, pues a pesar de que las agencias de evaluación y acreditación, así como otros actores relevantes que apoyan estas prácticas asumen una relación directa entre ambos conceptos, es importante reconocer que no se trata de sinónimos; y por otra parte, un grupo importante de figuras, entre las que destacan los propios académicos, cuestionan las prácticas de simulación ineludiblemente atadas al proceso.

Por su parte, las voces en favor de la acreditación mencionan entre los motivos para esta práctica los siguientes: 1) el autoestudio y reflexión que se deriva de los procesos de evaluación con miras a la acreditación que sin la urgencia de la acreditación tal vez no se propiciaría; 2) a la preferencia de la autorregulación por encima de la regulación por el Estado; 3) a la validación externa de los procesos de aseguramiento de la calidad; y 4) el proceso de revisión por pares permite una especie de 'fertilización cruzada' de ideas cuando las personas salen de su propio campus para realizar las visitas de evaluación (Council for Higher Education Accreditation [CHEA], 2006).

Los elementos a favor y en contra de la acreditación internacional llevaron a un intenso debate durante la Conferencia General de la UNESCO de 2003, y se llegó a la conclusión de que, tanto la evaluación de la calidad de la Educación Superior que imparten las IES, dentro de sus fronteras y en otros países, como la supervisión de los criterios y metodologías que se 
utilizan para llevar a cabo estos procesos, son elementos fundamentales para la valoración y prestigio internacionales del Sistema de Educación Superior de los países, lo que condujo a su vez a la elaboración de las 'Directrices en materia de calidad de la educación superior a través de las fronteras' con lineamientos destinados a todos los actores de la Educación Superior, de acuerdo con la UNESCO y la Organización para la Cooperación y el Desarrollo Económicos|(UNESCOOCDE, 2006). Este documento definió entonces que las IES "son responsables de la calidad y la pertinencia social, cultural y lingüística de la educación, así como de los parámetros de los diplomas otorgados en su nombre, independientemente del lugar o la manera como se imparte esa educación" (UNESCO-OCDE, 2006, p. 15) y exhorta a las IES a mantener o, en su caso elaborar, sistemas internos de gestión de calidad, a consultar y considerar las recomendaciones de los organismos evaluadores y acreditadores de la calidad, y a compartir sobre sus criterios y procedimientos para el aseguramiento de la calidad (UNESCO-OCDE, 2006).

El documento de directrices reconoce también la heterogeneidad de los organismos encargados de evaluar y acreditar la calidad de las IES, tanto al interior como al exterior de los países, la diversidad en los criterios, metodologías e instrumentos que estos utilizan para definir la calidad y el carácter de su participación en procesos de evaluación y acreditación. Por tanto, en este documento se insta a los organismos acreditadores a considerar la diversidad en la elaboración de sus criterios de evaluación, a crear y consolidar redes internacionales que permitan incrementar su capacidad para la evaluación de la calidad, a intercambiar información y conocer buenas prácticas en otros modelos, a facilitar la comprensión de otros sistemas de aseguramiento de la calidad, a ser transparentes en cuanto a los criterios y metodologías aplicadas en sus procesos de evaluación, y a participar en procesos de evaluación conjunta con otros organismos evaluadores (UNESCO-OCDE, 2006).

En cuanto a los modelos de evaluación con fines de acreditación internacional se puede decir que los más relevantes son el europeo y el americano. El primero se desarrolló a partir del proceso de Bolonia, promoviendo estándares compartidos, e inició sus actividades a través de agencias nacionales de evaluación con dinámicas institucionales y políticas nacionales, pero buscando una estandarización regional. Aunque existen agencias regionales organizadas por profesiones, se hace uso del principio de libre movilidad acordado por la Unión Europea, por lo que cada institución es libre de acreditarse con la agencia de su preferencia. Dentro de esta modalidad existe el European Consortium for Acreditation in Higher Education (ECAHE), un consorcio de 15 organizaciones nacionales de acreditación de 10 países, que también tienen la posibilidad de acreditar internacionalmente desde el año 2007 (Rama, 2009).

El modelo americano, por su parte, nace en 1888 con la creación de la Asociación de Colegios y Universidades de los Estados del Medio Oeste, que tenía como finalidad ayudar a las instituciones de esta región a elevar la calidad de sus programas y sobrevivir, pues la mayoría de los estudiantes optaban por las instituciones de mayor tradición ubicadas en la Costa Este; 
posteriormente surgieron una gran cantidad de agencias de acreditación, mismas que se agruparon por regiones, campos de conocimiento y profesiones (Viñas, 1991). Actualmente existen en Estados Unidos ocho agencias de evaluación y acreditación, organizadas por regiones, así como 81 organismos disciplinares que cuentan con reconocimiento para otorgar acreditaciones por parte del Council for Higher Education Accreditation (CHEA), y la mayoría de ellos realiza también acreditaciones en el extranjero (Rama, 2009).

Bauela-Casal, Vadillo, Pagani, Bermúdez, Sierra, Zych y Castro (2009) manifiestan que existen siete categorías de análisis para la evaluación con fines de acreditación internacional aceptadas por los diferentes organismos europeos y norteamericanos: programa educativo, organización de la enseñanza, recursos humanos, recursos materiales, el proceso educativo, resultados e investigación; de estas categorías se desprenden, en el caso de Estados Unidos, 11 indicadores, y para el caso de Europa un promedio de 23, ya que el número exacto varían en función del organismo y país.

\section{Las implicaciones de los procesos de evaluación y acreditación internacional en las IES}

Responder a los requerimientos que implican los procesos internacionales de aseguramiento de la calidad constituye una de las principales preocupaciones para las IES, porque de ello depende la posibilidad de tener acceso a recursos económicos, financiar las actividades que realizan en cumplimiento de sus funciones esenciales, y la posibilidad de posicionarse en el escenario internacional educativo. El embarcarse en un proceso de acreditación o evaluación internacional también es un gran reto, pues cuando una IES decide emprender este camino con seriedad y comprometerse con la mejora, la transformación de estructuras y prácticas tradicionales es un imperativo.

No obstante, si la decisión de participar en un proceso de evaluación o acreditación internacional no forma parte de una política institucional definida, en términos de un verdadero compromiso con el aseguramiento de la calidad, los riesgos de quedarse en el terreno de simulación son evidentes: el objetivo se corrompe, los beneficios privados priman sobre los sociales y se olvida que las prácticas de calidad constituyen un medio para diagnosticar la situación actual, emprender cambios y mejoras, y ser más pertinentes y socialmente responsables.

Para que una acreditación internacional signifique en verdad una garantía de calidad en una IES, debe realizarse un arduo trabajo en todos los procesos institucionales y con todos los actores involucrados; ese trabajo estará orientado a intensificar la planificación institucional, a fortalecer las funciones sustantivas de las IES y a inculcar una cultura de apertura al cambio y de aprendizaje colectivo. La administración y la gestión de la calidad son, en ese sentido, elementos fundamentales de la cultura de las IES "porque implica[n], y permea[n] a todas las personas, a los recursos, a los procesos y a los resultados" (Correa de Molina, 2004, p. 71). 
Existen dos dimensiones que, a nivel institucional, pueden aprovecharse para mejorar la calidad de las instituciones y potenciar su crecimiento y desarrollo, y que también son aplicables a los procesos internacionales de acreditación y evaluación: la calidad del liderazgo y la visión estratégica, y la secuencia de los planes y actividades previstos (Salmi, 2009):

- El liderazgo y la visión estratégica, atributos indispensables en quien ejerce la rectoría de una IES, son elementos fundamentales para llevar a cabo programas correctos y acordes con los objetivos de la institución; para identificar áreas específicas de ventaja comparativa que puedan aprovecharse para la proyección institucional, tanto nacional como internacionalmente; y para actuar con cautela en situaciones de incertidumbre.

- La correcta secuencia de intervenciones es fundamental en la planeación estratégica, que a su vez es una herramienta indispensable en la gestión. Dada la incesante competitividad en el ámbito internacional, las IES se ven presionadas a desarrollar estrategias que les permitan mantener o mejorar su posicionamiento y proyección; en ese sentido, estar atentos al contexto e identificar los cambios que están ocurriendo son factores fundamentales para realizar avances al interior de la institución y también para diseñar un plan estratégico que contenga una secuencia de actividades que permitan adaptarse a dichos cambios.

Es de notar que la evaluación es un elemento importante en estas dos dimensiones, pues permite a quien ejerce el rectorado y a su equipo de trabajo contar con insumos para la toma de decisiones pertinentes, conocer las fortalezas y debilidades de la institución, realizar una planeación estratégica basada en un diagnóstico de la situación actual, medir los avances en las metas propuestas, redefinir el rumbo en caso necesario, y a la vez "resistir la tentación de centrarse en un número limitado de acciones que estén directamente relacionadas con los indicadores específicos" (Salmi, 2009, p. 43), pues esto reduce el espectro de temas en los que se puede incidir.

El rector o rectora, como líder de una IES, debe ser quien promueva los procesos de mejora continua y aseguramiento de la calidad, y contagie este ímpetu de manera transversal y longitudinal en la organización, de tal manera que tanto el personal docente y administrativo, como el estudiantado, se apropien de dichos conceptos y los hagan parte de su actuar cotidiano. El o la titular de una IES debe, además, contar con una visión prospectiva, ser capaz de adelantarse a los cambios y emprender acciones ante los requerimientos del contexto. Se puede decir entonces que la tarea del líder de una IES es "definir y articular la misión de la institución, desarrollar objetivos significativos y luego reclutar el talento, construir un consenso, crear el clima y proveer los recursos para alcanzarlo" (Rhodes, 2009, p. 292), lo que aplica no solamente a los procesos de evaluación y acreditación internacional, sino a todo cambio o mejora organizacional que se pretenda emprender. 
Así, el primer paso para que se incluyan en la agenda institucional los procesos de aseguramiento de la calidad es la participación y el involucramiento del rector o rectora y su equipo, pues les corresponde a ellos liderar el proceso y mantenerlo en el largo plazo.

Las recomendaciones del Council for Higher Education Accreditation de los Estados Unidos resumen la importancia del liderazgo de la rectora o rector en la acreditación y consideran que su involucramiento debe iniciar antes del proceso de evaluación preparatorio y concluir tiempo después de que se superen las observaciones expresadas en los resultados de la visita de los evaluadores, es decir, durante todo el tiempo que tome llevar a cabo las mejoras en la institución; el compromiso de los máximos líderes de una institución con la acreditación, entendiendo este proceso como una forma de mejora, tiene un gran impacto en su éxito, pues su involucramiento es esencial en la definición de políticas institucionales y revisión de estándares y procedimientos. Las máximas autoridades de una IES deben dejar claro su respaldo al proceso a través de discursos y mensajes continuos, de tomar un papel activo en la organización de la evaluación diagnóstica y en la selección de los comités respectivos, y enfatizar su decisión de dar seguimiento a los resultados (CHEA, 2007).

Según Ferraro (2002) para que los procesos de aseguramiento de la calidad vayan más allá de la simulación y puedan integrarse en las prácticas cotidianas en el largo plazo, será necesario que estén impulsadas por líderes institucionales que tengan cuatro competencias básicas: a) mentalidad global, es decir, una perspectiva amplia de los temas, aprecio por los puntos de vista de los demás, flexibilidad y habilidades de pensamiento complejo; b) estabilidad personal, lo que implica mantener una postura abierta, una percepción aguda y una gran capacidad de resiliencia personal para afrontar las vicisitudes y sobreponerse al fracaso; c) confianza profesional para tomar decisiones, aunque estas a veces parezcan arriesgadas, y que sean capaces de balancear contradicciones sin priorizarlas o intentar eliminarlas; y d) liderazgo exitoso, que se traduce en la integración de equipos de trabajo diversos, comprometidos y entusiasmados con los retos que se les plantean.

Se reitera pues la importancia de que la implementación de procesos de aseguramiento de la calidad no se conciba como un fin en sí mismo, sino que se rescaten el valor de la autoevaluación y de la posibilidad de implementar mejoras (Correa de Molina, 2004), y se tome plena conciencia de que es el liderazgo de la rectoría el que puede movilizar a la institución y elevar sus estándares a partir de su participación en procesos de evaluación y acreditación. Un liderazgo fuerte y decidido reconoce que el mejoramiento de la calidad es un proceso continuo que nunca termina (CHEA, 2007).

\section{Conclusiones}

Esta revisión teórico-conceptual ha desatacado el papel de la evaluación y acreditación internacional en las IES y la participación del rectorado en los procesos conducentes. Como se ha mostrado, el rector o rectora de una IES es uno de los actores clave en dichos procesos 
pues de su liderazgo depende, en gran medida, la apropiación del compromiso con la calidad por parte de la organización en su conjunto. En los casos en que la cultura de la calidad es incipiente, la labor del rectorado en la gestión de un posible cambio organizacional en pro de una cultura de calidad a partir de la evaluación y acreditación es aún más relevante.

Las IES son organizaciones en las cuales los procesos de cambio son más complejos dadas las estructuras de poder y el conflicto de intereses que en ellas coexiste. La consideración de estos elementos revela con mayor claridad el protagonismo de quien ejerce la rectoría de una IES para liderar negociaciones de las que no solo se beneficien ciertos participantes sino la organización en su conjunto. Solamente con una activa participación de la persona que ejerce la máxima autoridad en una institución se puede lograr el involucramiento de todos los sectores y actores en las tareas orientadas al aseguramiento de la calidad en las funciones sustantivas de la IES y, por tanto, superar la posibilidad de la simulación y alcanzar un verdadero cambio organizacional enfocado al compromiso con la calidad.

Ante el panorama que presenta la evaluación y la acreditación internacional, parece indicado señalar que: 1) las IES no pueden abstenerse de participar en procesos de evaluación y acreditación internacional, pues esto es, cada vez con mayor frecuencia, un indicativo de su competitividad y legitimidad; 2) las IES que transitan adecuadamente por un proceso de evaluación o acreditación internacional obtienen, además del 'distintivo de calidad' y la percepción pública asociada a este, el valor añadido de una oportunidad para la reflexión sobre su misión, visión y quehacer educativo; y 3) para emprender un proceso de evaluación o acreditación internacional las IES requieren contar con líderes convencidos de sus beneficios y decididos a embarcarse en la encomienda.

Un rector o rectora que busca la implementación de una cultura de calidad a partir de emprender procesos de evaluación y acreditación internacional en su IES, deberá ejercer diferentes tipos de liderazgo:

a) En los pasos iniciales del proceso habrá que ejercer un liderazgo transformacional, con las habilidades técnicas, destrezas políticas, carisma y capacidad de consenso necesarios, y que además contagie de entusiasmo a los integrantes de su comunidad universitaria, compartiendo su visión y animándoles a participar en el proceso de evaluación y acreditación internacional.

b) El siguiente paso es ejercer un liderazgo trascendente, que permita a la rectoría contar con el compromiso ético y moral continuo de profesores, estudiantes y personal administrativo, de manera que el ímpetu inicial del proceso se mantenga durante sus fases más exigentes.

c) Apoyarse en todo momento con algunas dosis de liderazgo transaccional, lo que permitirá al rector o rectora hacer ver a todos los que colaboren en el proceso que su participación es apreciada, valorada y recompensada. 
Así, se puede concluir que un rector o rectora que decida embarcarse en un proceso de cambio y mejora de su organización, además de ejercer diversos tipos de liderazgo según el momento, deberá tener la capacidad de ejercer el poder eficaz y responsablemente, de comprender las motivaciones tan diversas que guían a los integrantes de su equipo inmediato y de otros miembros de la comunidad universitaria, y de inspirar y desarrollar una atmósfera propicia para la participación y el aprendizaje colectivos.

\section{Referencias}

Acosta Silva, A. (2010). Príncipes, burócratas y gerentes. El gobierno de las universidades en México. México, México: ANUIES-UDUAL.

Bauela-Casal, G., Vadillo, O., Pagani, R., Bermúdez, M. d., Sierra, J. C., Zych, I. \& Castro, A. (2009). Comparación de los indicadores de la calidad de las universidades RUSC. Universities and Knowledge Society Journal, 6(2), 9-21. Recuperado de: http://www.redalyc.org/ pdf/780/78012947008.pdf

Blanco-Ramírez, G. \& Berger, J. B. (2014). Rankings, accreditation and the international quest for quality: organizing an approach to value in higher education. Quality Assurance in Education, 22(1), 88104. Recuperado de: http://www.emeraldinsight.com/doi/abs/10.1108/QAE-07-2013-0031

Bolívar, A. (1997). Liderazgo, mejora y centros educativos. En: Medina, A. (coord.). El liderazgo en educación. Madrid: UNED. Recuperado de: http://www.textosescolares.cl/usuarios/ convivencia escolar/doc/201103070200190.U\%20de\%20Granada.Liderazgo Mejora y Centros Educativos.pdf

En A. Medina (coord.): El liderazgo en educación. (pp. 25-46). Madrid: UNED.

Botero Chica, C. A. (2008). Cinco tendencias de la gestión educativa. Revista lberoamericana de Educación, 49(2), 1-11. Recuperado de: http://www.rieoei.org/deloslectores/2811Botero.pdf Council for Higher Education Accreditation (CHEA). (2006). Presidential perspectives on accreditation: a report of the CHEA Presidents project. Washington: Institute for Research and Study of Accreditation and Quality Assurance. Recuperado de: http://www.chea.org/ pdf/CHEAMonograph Apr06.pdf

Council for Higher Education Accreditation (CHEA). (2007). Presidential guidelines series, 1, 1-6. Washington, DC: Council for Higher Education Accreditation. Recuperado de: http://www. chea.org/pdf/PrezGL1.pdf 
Correa de Molina, C. (2004). Gestión y evaluación de la calidad en la educación. Bogotá: Cooperativa Editorial Magisterio.

Ferraro, G. P. (2002). The cultural dimension of international business. New Jersey: Prentice Hall.

Global University Network for Innovation (GUNI). (2009). La educación superior en tiempos de cambio. Nuevas dinámicas para la responsabilidad social: Síntesis de los informes GUNI la educación superior en el mundo. Madrid: Mundi-Prensa.

Held, D., McGrew, A., Goldblatt, D. \& Perraton, J. (2000). The Global Transformations Reader. Inglaterra: TJ International. Recuperado de: https://throughtimesandspace.files. wordpress.com/2013/10/presentasi-the-global-transformations-reader-an-introductionto-the-globalization-debate-eds-david-held-anthony-mcgrew.pdf

Jiménez, G. (1999). Evaluación del estado de salud con la encuesta SF-36: Resultados preliminares en México. Salud Pública de México, 41(2), 110-118. Recuperado de: http://www.scielosp. org/scielo.php?script=sci arttext\&pid=S0036-36341999000200005

Knight, J. \& De Wit, H. (1999). Quality and Internationalisation in Higher Education. Paris: OECD, IMHE. Koontz, H. \& Weihrich, H. (1998). Administración, una perspectiva global. México: McGraw-Hill.

Madrigal T., B. E. (2013). Líderes y Liderazgo. Guadalajara, México: Editorial Universitaria Universidad de Guadalajara.

Palomino Vadillo, M.T. (2013). Liderazgo y motivación en equipos de trabajo. Madrid: ESIC Business \& Marketing School.

Rama, C. (2009). El nacimiento de la acreditación internacional. Avaliação: Revista da Avaliação da Educação Superior, 14(2), 291-311. Recuperado de: http://www.scielo.br/scielo. php?script=sci arttext\&pid=S1414-40772009000200004

Rama, C. (2012). La acreditación internacional. (III Congreso Internacional CACECA, Agencias internacionales de acreditación, retos y oportunidades). Puerto Vallarta: CACECA.

Rhodes, F. H. (2009). La creación de futuro: la función de la universidad norteamericana. Buenos Aires: Universidad de Palermo.

Robbins, S. P. (2004). Comportamiento organizacional. USA: Pearson Educación.

Salmi, J. (2009). El desafío de crear universidades de rango mundial. Washington: Banco Mundial en coedición con Mayol Ediciones S.A. 
Sebastián, J. (2003). La dimensión internacional en los procesos de evaluación y acreditación de la educación superior. En: Educación Superior, calidad y acreditación. Bogotá: Consejo Nacional de Acreditación de Colombia.

Sanyal, B. y Martin, M. (2009). Garantía de la calidad y el papel de la acreditación: una visión global. En: La Educación Superior en tiempos de cambio. Nuevas dinámicas para la responsabilidad social. Recuperado de: http://upcommons.upc.edu/handle/2099/7499

Organización de las Naciones Unidas para la Educación, la Ciencia y la Cultura y Organización para la Cooperación y Desarrollo Económicos (UNESCO-OCDE). (2006). Directrices en materia de calidad de la educación superior a través de las fronteras. París: UNESCO.

Uribe B., M. (2007). Liderazgo y competencias directivas para la eficacia escolar: Experiencia del Modelo de Gestión Escolar de Fundación Chile. Revista Electrónica Iberoamericana sobre Calidad, Eficacia y Cambio en Educación, 5(5e), 149-156. Recuperado de: http://www. redalyc.org/pdf/551/55121025022.pdf

Van der Wende, M. (1997). Internationalising the curriculum in Dutch higher education: An international comparative perspective. Journal of studies in internationaleducation, 1(2), 53-72. Recuperado de: http://jsi.sagepub.com/content/1/2/53.abstract

Viñas, J. (1991). La universidad norteamericana, el autoestudio y la acreditación: un modelo de mejoramiento académico. Revista de la Educación Superior, (79), 21-35. Recuperado de: http://publicaciones.anuies.mx/pdfs/revista/Revista79 S1A2ES.pdf 